\title{
Die «Arisierung» von jüdischem Grundeigentum während des National- sozialismus: ein Fallbeispiel aus Berlin - Prenzlauer Berg
}

\section{Daniela Zunzer, Basel}

\section{Einleitung}

Nach der Vereinigung der beiden deutschen Staaten im Jahr 1990 und im Zuge des Übergangs von der sozialistischen Planwirtschaft zur Marktwirtschaft wurden die Eigentumsverhältnisse in den neuen Bundesländern neugeordnet. Der in der DDR weitgehend verstaatlichte Grund und Boden wurde auf der Grundlage des Vermögensgesetzes in Privateigentum zurücküberführt. Ziel der vermögensrechtlichen Regelungen war es zunächst, «unredliche» Verkäufe und Enteignungen aus der Zeit der DDR wiedergutzumachen. Frühere Eigentümer erhielten ihre Grundstücke zurück, wenn sie ohne oder mit einer zu geringen Entschädigung enteignet worden waren (GLOcK \& Keller 2001: 95). Die DDR hatte sich im Gegensatz zur Bundesrepublik der Wiedergutmachung nationalsozialistischen Unrechts auf ihrem Terrain weitgehend widersetzt. Dieser Umstand war die moralisch mehr als gerechtfertigte, rechtlich jedoch nicht zwingend erforderliche Begründung für die Ausdehnung der vermögensrechtlichen Regelungen auf die Eigentumsentziehungen aus der Zeit von 1933 bis 1945 (REIMANN 2000: 19-21). Betroffen von diesen während des Nationalsozialismus stattgefundenen rechtswidrigen Eigentumsübergängen waren vor allem jüdische Eigentümer.

Allein in Berlin wurden Anfang der 90er Jahre ca. 175.000 Anträge auf Rückübertragung gestellt, davon ca. 35.000 in bezug auf verfolgungsbedingten Vermögensverlust zwischen 1933 und 1945 (REIMANN 2000: 33). Die gründerzeitlichen innerstädtischen Altbauten waren nahezu flächendeckend restitutionsbehaftet. Für viele dieser Grundstücke lagen konkurrierende Anträge verschiedener ehemaliger Eigentümer vor. Hatten auf einem Grundstück mehrere rechtswidrige Transfers in Folge stattgefunden, wurde das Haus an den zeitlich zuerst Geschädigten zurückgegeben, die übrigen berechtigten Anspruchsteller erhielten eine Entschädigung. In Vorbereitung der Rückgabe an die privaten Eigentümer musste die Eigentumsgeschichte der Grundstücke in vielen Fällen bis 1933 zurückverfolgt werden.

Im Zusammenhang damit gewann ein Aspekt der nationalsozialistischen Herrschaft wieder an Aktualität und zwar die "Arisierung» des jüdischen Grundeigentums, d.h. die systematische Erfassung sowie die umfassende Benachteiligung bis hin zur Enteignung jüdischer Grundeigentümer. Im Vergleich zu den schon seit längerem detaillierter erforschten "Arisierungen» von Einzelhandel und Gewerbe wurde die "Arisierung» von Immobilien bislang in der Forschung eher am Rande berücksichtigt. Erst in den letzten Jahren sind neuere Forschungsarbeiten und Ausstellungen dazu entstanden (vgl. HistoriKerKommission (Hrsg.) 2000a, 2000b; «Betrifft: Aktion 3» 1998, GRUNER 2000).

Ziel des vorliegenden Textes ist es, die rechtlichen Regelungen und den Ablauf der "Arisierung» des Grundeigentums für den gesamten Zeitraum der NSHerrschaft im Überblick darzustellen und mit einer lokalen Fallstudie den Prozess der «Arisierung» für einen konkreten städtischen Teilraum aufzuarbeiten.

Diese Darstellung trägt dazu bei, einzelne «Arisierungen» in den Gesamtzusammenhang der systematischen Benachteiligung und Verfolgung bis hin zur Vernichtung der jüdischen Bevölkerung einordnen zu können und gibt einen Überblick über das Ausmass und den zeitlichen Verlauf der «Arisierung» in einem konkreten Gebiet.

Weiterhin kann die Aufarbeitung der Vorgänge, durch die bestimmte Bevölkerungsgruppen ihr Eigentum (in unterschiedlichen politischen Systemen) verloren haben, einen Beitrag dazu leisten, die Naturalrestitution als ethisch und moralisch gerechtfertigten Versuch der Wiedergutmachung zu sehen.

Im ersten Teil des Textes werden die gesetzlichen Regelungen und die Durchführung der "Arisierung» beschrieben. Dafür wurde die aktuelle Forschungsliteratur erfasst. Im zweiten empirisch ausgerichteten Teil wird am Beispiel eines Teilgebiets des Ostberliner Stadtbezirks Prenzlauer Berg aufgezeigt, in welchem Umfang jüdisches Grundeigentum dort existierte und wie die «Arisierungen» dort durchgeführt wurden.

\section{Die «Arisierung» des jüdischen Grundeigentums}

\subsection{Immobilienmarktentwicklungen der Weimarer Republik}

Der gründerzeitliche Altbaubestand Berlins befand sich vor der Inflation zum Grossteil im Eigentum kleinerer Gewerbetreibender, Selbständiger und Handwerker, die ihr Haus oft als finanzielle Absicherung im Alter benutzten. Mit der Inflation 1922/23 und der Wirtschaftskrise ging eine weitgehende Veränderung der Eigentümerstruktur einher. 


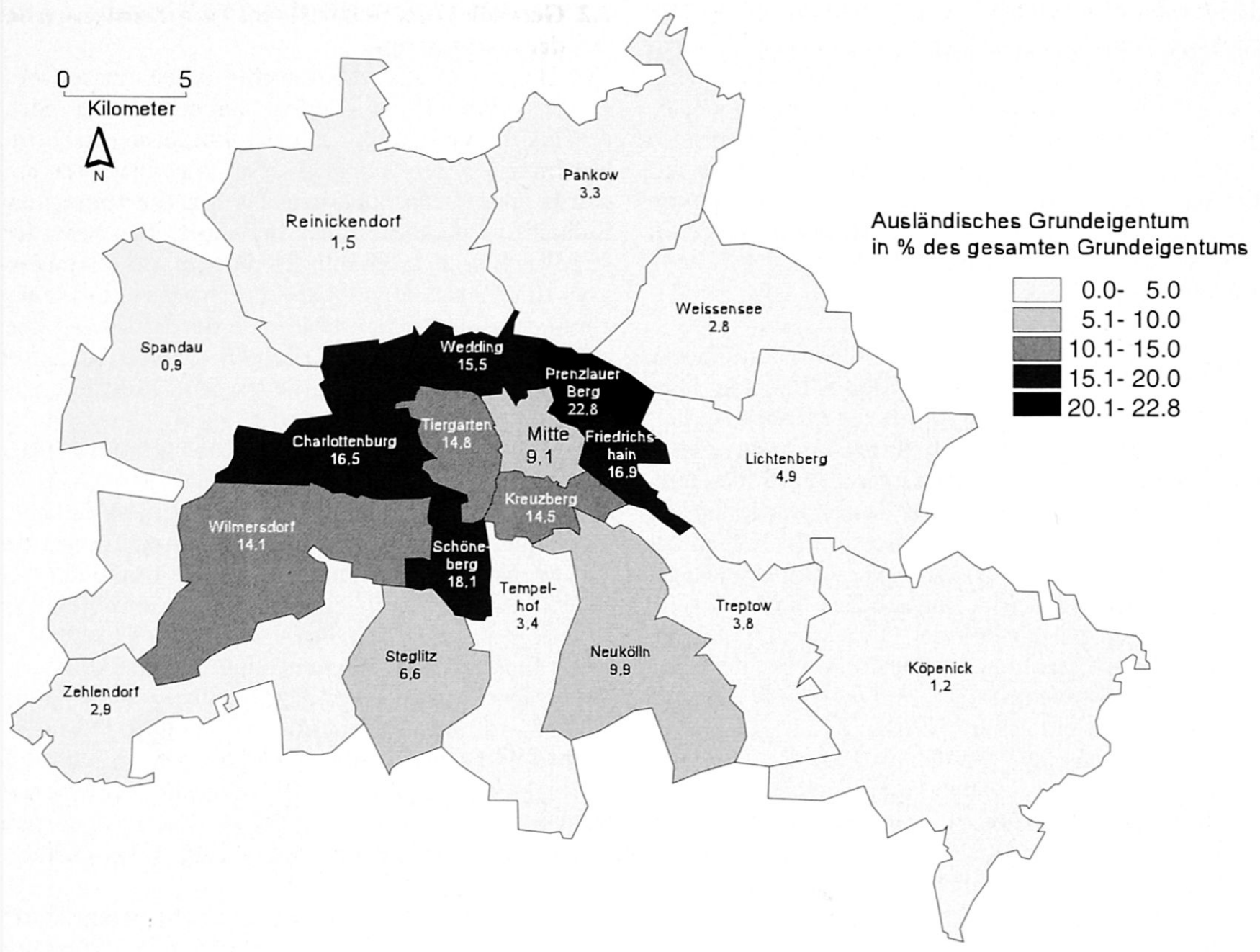

Abb. 1: Grundstücke im Eigentum von Personen mit ausländischer Staatangehörigkeit in Berlin 1927 (in Prozent der Gesamtzahl der bebauten Grundstücke)

Real estate property of persons with foreign citizenship in Berlin, 1927 (as a percentage of the total of real estate property) Popriété foncière appartenant à des personnes de nationalité étrangère à Berlin (1927) en pourcentage du total de l'espace construit

Quelle:Preussische Statistik, Bd. 299; Wohnungszählung 1927:71; Kartographie: Entwurf und Bearbeitung: D. ZunZER

- Tendenziell führte der rapide Wertverlust der Reichsmark seit Anfang der 20er Jahre zur Flucht in Sachwerte als sicherere Geldanlagemöglichkeit und damit zu einer erhöhten Nachfrage nach Immobilien.

- Hingegen sahen sich jene Eigentümer, die über die Immobilien hinaus nur wenig finanzielle Reserven hatten, in vielen Fällen zum Verkauf gezwungen, um überhaupt noch den eigenen Lebensunterhalt finanzieren zu können. Dadurch entstand ein entsprechendes Angebot an Immobilien und die Kaufpreise sanken.

- Vor allem Investoren, die über stabile, d.h. ausländische Währungen verfügten, konnten zu günstigen Konditionen Immobilien erwerben. Als Reaktion darauf war 1923 eine Genehmigungspflicht für Grundeigentumserwerb durch Ausländer eingeführt worden, die aber umgangen werden konnte, wenn das Grundeigentum in eine Grundstücksgesellschaft in Form einer $\mathrm{AG}$ oder $\mathrm{GmbH}$ eingebracht wurde (ZUNZER 1996: 55ff.).

- Nach der Währungsstabilisierung waren die Wiederverkäufe der Immobilien sehr gewinnversprechend. Das führte erneut zu einem erhöhten Verkaufsgeschehen (Das Grundeigentum 1934: 945).

Die Eigentumstransfers der Inflationszeit haben in Berlin zu einer Internationalisierung der Eigentümerstruktur geführt. In der Reichswohnungszählung vom Mai 1927 wurden umfassende Daten über die Grundeigentümerstruktur erhoben (erfasst wurden aber nur Grundstücke im Eigentum natürlicher Personen). Danach befanden sich $8,8 \%$ der bebauten Grundstücke Berlins in ausländischem Eigentum, $92 \%$ davon hatten 
die Immobilien nach dem 1.7.1918 erworben. Die höchsten Anteile verzeichneten die Innenstadtbezirke Berlins (Mitte, Tiergarten, Wedding, Prenzlauer Berg, Friedrichshain, Kreuzberg) sowie die westlichen Stadtbezirke Charlottenburg, Wilmersdorf und Schöneberg (vgl. Abb.1). In Berlin stellten Polen den grössten Teil ausländischer Grundeigentümer $(23,2 \%)$, gefolgt von Österreichern (17,5\%), Tschechoslowaken (10,7\%), Russen $(9,5 \%)$ und Spaniern (6,5\%) (Zunzer 1996: $60)$.
2.2 Gesetzliche Regelungen und Vorgehensweisen bei der «Arisierung»

Der Begriff «Arisierung» tauchte bereits in der Weimarer Republik im Umfeld antisemitischer Strömungen in Verbindung mit der Forderung nach der Verdrängung der Juden aus der Wirtschaft auf und wurde von den Nationalsozialisten übernommen und radikalisiert (BAJOHR 2000: 15). Auch die ausländerfeindliche und antisemitische Polemik einer angeblichen «Überfremdung» des Grundeigentums hatte

\begin{tabular}{|c|c|}
\hline 30.1.1933 & Machtübernahme durch die Nationalsozialisten \\
\hline 14.7.1933 & $\begin{array}{l}\text { Gesetz über den Widerruf von Einbürgerungen und die } \\
\text { Aberkennung der deutschen Staatsangehörigkeit } \\
\text { (richtete sich in erster Linie gegen die nach } 1918 \text { eingebürgerten Juden aus } \\
\text { den damaligen deutschen Ostgebieten; nach einer Ausbürgerung verfiel das } \\
\text { gesamte inländische Vermögen dem Deutschen Reich) }\end{array}$ \\
\hline 15.9.1935 & $\begin{array}{l}\text { Reichsbürgergesetz } \\
(\text { (Nürnberger Gesetze») }\end{array}$ \\
\hline 26.4.1938 & $\begin{array}{l}\text { Verordnung über die Anmeldung aller jüdischen Vermögen } \\
\text { über 5000 RM } \\
\text { (Anmeldung des gesamten in- und ausländischen Vermögens bis zum } \\
\text { 30.6.1938) }\end{array}$ \\
\hline 14.6.1938 & $\begin{array}{l}\text { 3. Verordnung zum Reichsbürgergesetz } \\
\text { (Festlegung bestimmter Gewerbebetriebe und Geschäftsformen als } \\
\text { «jüdische im Sinne des Reichsbürgergesetzes») }\end{array}$ \\
\hline 9./10.11.1938 & $\begin{array}{l}\text { Novemberpogrom } \\
\text { («Reichskristallnacht»; Zerstörung von Synagogen, Geschäften und } \\
\text { Wohnhäusern) }\end{array}$ \\
\hline 12.11.1938 & $\begin{array}{l}\text { Verordnung zur Ausschaltung der Juden aus dem deutschen } \\
\text { Wirtschaftsleben } \\
\text { (Schliessung aller jüdischen Geschäfte zum 1.1.1939 angeordnet) }\end{array}$ \\
\hline 3.12 .1938 & $\begin{array}{l}\text { Verordnung über den Einsatz jüdischen Vermögens } \\
\text { (Schaffung der rechtlichen Grundlage für die «Zwangsarisierung» des } \\
\text { gesamten jüdischen Vermögens) }\end{array}$ \\
\hline 30.4.1939 & $\begin{array}{l}\text { Gesetz über Mietverhältnisse mit Juden } \\
\text { (Gesetzliche Vorbereitung zur Zusammenlegung jüdischer Familien in sog. } \\
\text { «Judenhäusern»; Aufhebung des Räumungsschutzes u.ä. für jüdische } \\
\text { Mieter) }\end{array}$ \\
\hline 17.9.1940 & $\begin{array}{l}\text { Verordnung über das Vermögen von Angehörigen des ehemals polnischen } \\
\text { Staates } \\
\text { (Beschlagnahme v.a. jüdischer Vermögenswerte durch die } \\
\text { Haupttreuhandstelle Ost - HTO) }\end{array}$ \\
\hline 25.11.1941 & $\begin{array}{l}\text { 11. Verordnung zum Reichsbürgergesetz } \\
\text { (Einziehung des gesamten jüdischen Vermögens bei der Deportation; } \\
\text { Verfall an das Deutsche Reich) }\end{array}$ \\
\hline
\end{tabular}

Tab. 1: Wichtigste rechtliche Regelungen im Hinblick auf die «Arisierung» des Grundeigentums Most important legal regulations concerning the «aryanization» of real estate property Les dispositions légales les plus importantes concernant «l'aryanisation» de la propriété foncière Quellen: SCHEFFLER 1964: 89-91; ZUNZER 1996 
bereits Ende der 20er Jahre eingesetzt (AlbRECHT 1930: 732).

Ziel der nationalsozialistischen "Arisierungspolitik» war die vollständige Verdrängung der Juden aus der Wirtschaft, d.h. aus jeglicher selbständiger Wirtschaftstätigkeit und gleichzeitig die Bereicherung von staatlichen Stellen durch ein sukzessive umfassenderes Einbehalten von jüdischen Vermögenswerten (vgl. hierzu auch Gruner 2000). Die Wirtschaftstätigkeit unterteilte sich im Sinne der hierzu erlassenen Gesetze und Regelungen in Gewerbebetriebe und Einzelhandelsunternehmen einerseits sowie in Grundeigentum andererseits. Das Ergebnis der "Arisierung» war «eine der grössten Besitzumschichtungen der Neuzeit» (VAN LAAK 1992: 232).

Während der nationalsozialistischen Herrschaft sind unter «Arisierung» nicht nur jene Vorgänge zu verstehen, bei denen die jüdischen Grundeigentümer einer direkten, persönlichen Verfolgung ausgesetzt waren und dadurch ihr Eigentum und Vermögen verloren, sondern alle Eigentumsübergänge, die kausal mit den gesamten politischen und wirtschaftlichen Rahmenbedingungen des Nationalsozialismus im Zusammenhang stehen.

Nicht unter die «Arisierung» fällt die ebenso systematische Verdrängung von Juden aus Angestellten- und Beamtentätigkeiten und ebenso wenig die im nationalsozialistischen Jargon als «Entjudung» bezeichnete Vertreibung von jüdischen Mietern aus ihren Wohnungen. In Berlin oblag diese mit ebenso grosser bürokratischer Akribie durchgeführte Aufgabe dem Generalbauinspektor SPEER, der v.a. zuständig war für die Neuplanung Berlins als «Reichshauptstadt Germania».

In fast allen Bereichen ihrer Herrschaft versuchten die Nationalsozialisten, ihr Tun mittels gesetzlicher Erlasse zu sanktionieren und somit zu einem quasi legitimierten Verwaltungsakt zu machen. Auch die «Arisierung» der deutschen Wirtschaft wurde vordergründig mittels Gesetzen und Verordnungen gehandhabt. Der Ablauf der "Arisierung» lässt sich auf der Grundlage dieser Regelungen grob in drei Phasen einteilen. Die erste Phase der "Arisierung» bis 1938 betraf v.a. das Einbehalten von Vermögenswerten im Falle der Emigration. In der zweiten Phase von 1938 bis 1941 wurden systematisch jüdische Vermögenswerte erfasst und jüdische Unternehmen «zwangsarisiert». Die letzte Phase der "Arisierung» ab 1941 war geprägt von Enteignungen jüdischer Grundeigentümer im Zusammenhang mit deren Deportation in die Vernichtungslager.

\section{«Freiwillige Arisierungen» - 1933 bis 1938}

Bis 1938 gab es keine gesetzliche Handhabe, jüdische Unternehmen, Immobilien und andere Vermögenswerte zwangsweise in «arisches» Eigentum zu überführen. Die Nationalsozialisten setzten in diesen Jahren vorwiegend auf sogenannte «freiwillige Arisierungen». Doch bezog sich das Wort «freiwillig» lediglich darauf, dass die jüdischen Eigentümer überhaupt noch selber tätig werden konnten und die Möglichkeit hatten, mit den Käufern ihres Eigentums zu verhandeln (GENSCHEL 1966: 127).

Alle Verkäufe und Versteigerungen sind vor dem Hintergrund einer zunehmenden Benachteiligung und Verfolgung der jüdischen Bevölkerung zu sehen. Bereits in den ersten Jahren des NS-Regimes wurden jüdische Grundeigentümer gegenüber nichtjüdischen zunehmend benachteiligt. Mittels politischem, wirtschaftlichem und ideologischem Druck sollten die jüdischen Eigentümer in eine Position gebracht werden, in der ihnen nichts weiter übrig blieb, als ihr Eigentum zu den diktierten Bedingungen zu veräussern. Gläubiger hoch dotierter Hypotheken konnten durch die kurzfristige Kündigung von Grundstücksbeleihungen die Zahlungsunfähigkeit des Hauseigentümers herbeiführen und eine Zwangsversteigerung erwirken. Nicht selten fungierten die Geldgeber dann selbst als Ersteigerer. Viele jüdische Eigentümer versuchten sich rechtlich dagegen zu wehren, jedoch mit immer geringerem Erfolg (BARKAI 1988: 75). Es ist zu vermuten, dass das Wissen um die jüdische Identität des Verkäufers für diesen nachteilige Folgen hatte, da der potentielle Käufer den Verkaufsdruck ausnutzen konnte, um etwa den Kaufpreis zu drücken. Welchen Anteil des Kaufpreises der jüdische Eigentümer dann tatsächlich erhalten hat und inwiefern er darüber frei verfügen konnte, ist in vielen Fällen sehr schwer rekonstruierbar. Angesichts der kollektiven Verfolgung der jüdischen Bevölkerung seit Januar 1933 wurde bei der Restitution davon ausgegangen, dass es sich auch bereits bei den sogenannten Frühverkäufen bis September 1935 um Zwangsverkäufe handelte (REIMANN 2000: 34).

Die gesetzlichen Massnahmen der ersten Jahre der NS-Herrschaft bis Ende 1937 sanktionierten den zunehmenden staatlichen Zugriff auf persönliche Vermögenswerte im Falle einer Auswanderung aus dem Deutschen Reich,zum Teil in Fortführung entsprechender Gesetzgebung aus der Weimarer Republik. In der Endphase der Weimarer Republik waren als Folge der Wirtschaftskrise Gesetze mit dem Ziel einer Verhinderung der Kapitalflucht aus Deutschland erlassen worden, die auch nach 1933 ihre Gültigkeit weiterhin besassen. So war 1931 die sogenannte «Reichsfluchtsteuer» eingeführt worden. Bei Aufgabe des Wohnsitzes im Deutschen Reich mussten 25\% des Vermögens und Einkommens an den Staat abgeführt werden. Das Restvermögen wurde auf ein Auswanderersperrkonto überwiesen. Der Umtausch in Devisen bedeutete mit Wechselkursen in Höhe von $50 \%$ des offiziellen Kurses im Jahre 1935 bis hin zu 4\% im Jahre 1939 dramatische 
Verluste für die Emigranten. Grundsätzlich erstreckte sich der Geltungsbereich dieser Regelungen zwar auf alle Reichsangehörigen, doch war die jüdische Bevölkerung durch die nationalsozialistische Verfolgung und durch eine sie benachteiligende Auslegung der Gesetze überdurchschnittlich häufig davon betroffen (Einige Dokumente 1954: 40-42; BarKaI 1988: 111f.). Aus der Emigration der von ihnen verfolgten Bevölkerungsgruppen zogen die Nationalsozialisten zunehmende Gewinne ab, indem sie bis 1938 alle alternativen Möglichkeiten, Vermögen ausser Landes zu bringen, etwa mittels Tausch von Grundstücken oder Beteiligung an ausländischen Unternehmen, sukzessive verboten (HILbERG 1990: 142).

\section{Gezielter Vermögenszugriff ab 1938}

In der zweiten Phase der «Arisierung» seit Anfang des Jahres 1938 wurden Gesetze und Erlasse veröffentlicht, die gezielt die unternehmerische Grundlage der jüdischen Bevölkerung vernichten sollten und einen direkten Zugriff auf jüdische Vermögenswerte ermöglichten. Nach Erlass der Anmelde- und der Einsatzverordnung vom April und Dezember 1938 (vgl. Tab. 1) hatte der NS-Staat Ende des Jahres 1938 das gesamte jüdische Vermögen, insofern es nicht von einem anderen Staat geschützt wurde, erfasst und unter Kontrolle gebracht.

Damit waren alle Vorbereitungen für eine «Zwangsarisierung» getroffen. Jüdische Immobilieneigentümer hätten ab Ende 1938 zum Verkauf ihrer Häuser gezwungen werden können. Doch die Verkaufswelle, die nach der Pogromnacht vom 9.11.1938 von Seiten jüdischer Eigentümer einsetzte, zeigte aus nationalsozialistischem Blickwinkel unerwünschte Folgen (WALK 1981: 259, Genschel 1966: 144): Es bestünde die Gefahr, dass «der Erwerb der Grundstücke von unanständigen Elementen ausgenutzt wird, da die zum Wehrdienst eingezogenen Personen sich nicht hierbei beteiligen können» (Deutsche WohnwirTsChaft 1940: 245). Daher wurde die Weisung erlassen, eine zwangsweise «Arisierung» des Grundeigentums bis auf weiteres zu unterlassen. Im Gegensatz dazu wurden alle jüdischen Unternehmen und Gewerbebetriebe zum 1.1.1939 «zwangsarisiert».

Die Grundstücksverkäufe von Seiten jüdischer Eigentümer wurden allerdings vollständig kontrolliert und reglementiert:

- Alle Rechtsgeschäfte jüdischer Verkäufer bedurften einer gesonderten behördlichen Genehmigung.

- Über diese Genehmigung konnte Einfluss auf die Auswahl des Käufers ausgeübt werden, und gleichzeitig wurde von staatlicher Seite stärker regulierend in die Kaufpreisentwicklung eingegriffen jedoch kaum zugunsten der jüdischen Eigentümer, sondern eher mit Blick auf staatliche Einnahmequellen.
- Überschritt der Kaufpreis die behördlichen Vorgaben, wurde er für den jüdischen Verkäufer herabgesetzt. Galt der vereinbarte Kaufpreis als zu niedrig, wurde er für den Käufer angehoben. Die Differenz zwischen dem Preis, den der «arische» Erwerber zahlte und dem, den der jüdische Verkäufer erhielt, wurde als "Arisierungsabgabe» vom Staat eingezogen (Brombach 1941: 216, Scholl 1940: 26ff.).

- Die Kaufpreisprüfung bezweckte somit zwei Dinge: zum einen sollte der jüdische Verkäufer so wenig wie möglich für sein Grundstück erhalten, zum anderen sollte der Staat, nicht der Erwerber, durch die Einziehung der «Arisierungsabgabe» profitieren.

Eine «Zwangsarisierung» des Grundeigentums scheint vor allem aus zwei Gründen aufgeschoben worden zu sein. Der Erwerb des bis anhin noch nicht veräusserten jüdischen Grundeigentums sollte den besonders verdienten "Volksgenossen», den an der Front kämpfenden Wehrmachtsoldaten, nach ihrer Rückkehr vorbehalten sein. Ein zweiter Grund für einen Aufschub der «Zwangsarisierung» von jüdischem Grundeigentum war die Überlegung zu einer «Ghettoisierung» der Juden in sogenannten «Judenhäusern» - d.h. Häusern jüdischen Eigentums (AdLER 1974: 44). In erster Linie war diese Zusammenlegung als Vorbereitung für die anschliessende Deportation geplant gewesen (WISDALSKI 1989: 17).

\section{Enteignung und Verfall ab 1940}

Ab Ende des Jahres 1940 begann mit den Beschlagnahmungen von jüdischem Vermögen in den okkupierten Gebieten die letzte Phase der «Arisierung». Das Vermögen (Unternehmens-, Immobilien- und Geldvermögen) von jüdischen Angehörigen jener Staaten, die nach der Okkupation dem Deutschen Reich eingegliedert wurden, wurde nahezu identisch wie das Vermögen von Juden deutscher Staatsangehörigkeit behandelt. Besonders rigoros war die Vorgehensweise im eingegliederten Teil Polens mit Einsetzen der Haupttreuhandstelle Ost (HTO) im Herbst 1940. Das Vermögen aller polnischen Staatsangehörigen, die als nicht «eindeutschungsfähig» erachtet wurden - vorwiegend Polen jüdischer Herkunft - wurde systematisch beschlagnahmt. Für die übrigen okkupierten Gebiete wurden jeweils unterschiedliche, in ihrer Stossrichtung aber vergleichbare Regelungen erlassen.

Eine letzte Verschärfung der Gesetzgebungstätigkeit im Hinblick auf die «Arisierung» des Grundeigentums im sogenannten «Altreich» erfolgte 1941. Mit der 11. Verordnung zum Reichsbürgergesetz wurde geregelt, dass das Vermögen der in die Vernichtungslager nach Osten deportierten Juden dem Deutschen Reich verfiel (Adler 1974: 628). Nach Erlass der 11. Verordnung war für Juden ein Verkauf ihres Eigentums praktisch unmöglich. Abgeschlossene Kaufverträge wurden 
nicht mehr genehmigt. Bei den Grundstücken wurde nach dem Verfall das Deutsche Reich im Grundbuch als Eigentümer eingetragen (WALK 1981:372). Die Verwaltung und «Verwertung» der verfallenen Vermögen oblag laut der Verordnung dem Oberfinanzpräsident (OFP) Berlin in Zuständigkeit für das ganze Reichsgebiet (ADLER 1974: 510).

Im Gegensatz zu den Gewerbebetrieben in jüdischem Eigentum, die zum 1.1.1939 zwangsweise «arisiert» wurden, hatte es bis zum Ende der nationalsozialistischen Herrschaft keine derartige Verfügung zur «Zwangsarisierung» des jüdischen Grundeigentums gegeben. Die jüdischen Eigentümer, die bis Ende der 30er Jahre dem ökonomischen und politischen Druck zu verkaufen noch standgehalten hatten und denen es nicht mehr gelang zu fliehen, wurden jedoch mit ihrer Deportation enteignet. Ihr gesamtes Eigentum fiel dem Deutschen Reich zu.

\section{Entstehung und Vernichtung jüdischen Grundeigen- tums am Helmholtzplatz, Berlin - Prenzlauer Berg}

\subsection{Methodisches Vorgehen}

Der Berliner Innenstadtbezirk Prenzlauer Berg wurde im Rahmen des HoBRECHTschen Bebauungsplanes von 1862 vorwiegend zwischen 1870 und 1920 mit mehrgeschossigen Mietshäusern bebaut und ist heute einer der am dichtesten besiedelten Bezirke Berlins. Zusammen mit dem Nachbarbezirk «Mitte» wies er ausgehend von den gestellten Restitutionsanträgen Anfang der 90er Jahre in Ostberlin die höchsten Anteile vermutlich ehemals jüdischen Eigentums auf. Empirisch untersucht wurde hier die Gegend um den Helmholtzplatz (vgl. Abb. 2).

Zunächst wurde unter Zuhilfenahme verschiedenster Quellen eine Liste mit Grundstücken zusammenge-

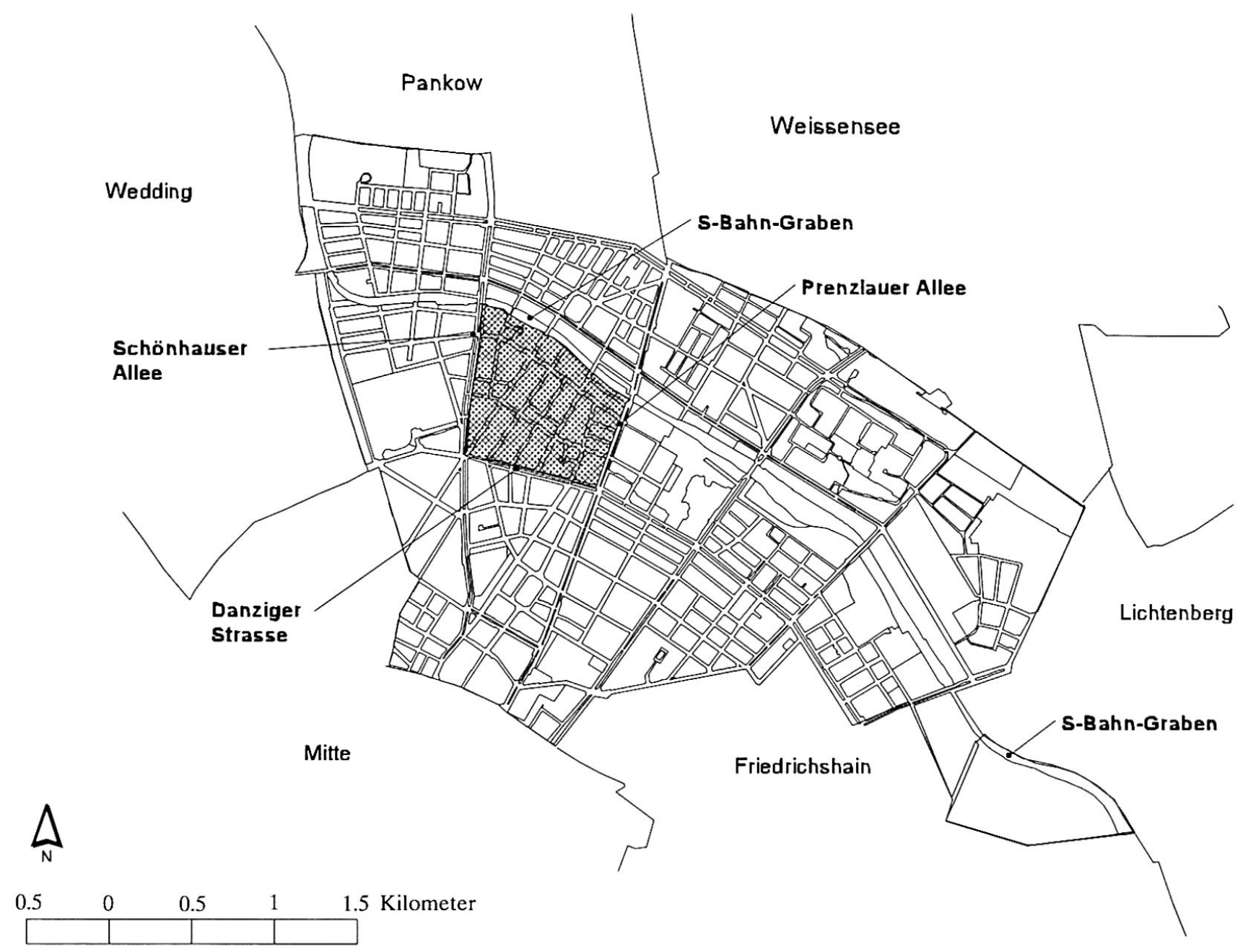

Abb. 2: Untersuchungsgebiet Helmholtzplatz in Berlin-Prenzlauer Berg Investigation area Helmholtzplatz in Berlin-Prenzlauer Berg Champ d'investigation Helmholtzplatz à Berlin-Prenzlauer-Berg Kartengrundlage Statistisches Landesamt BerLin; Kartographie und Bearbeitung: D. Zunzer 
stellt, die sich aller Wahrscheinlichkeit nach 1933 in jüdischem Eigentum befanden. Dafür wurden die Eigentümernennungen der Berliner Adressbücher mit dem Jüdischen Adressbuch Berlin abgeglichen. Darin sind jedoch ausserhalb von Berlin lebende jüdische Eigentümer und diejenigen, die nicht Mitglieder der jüdischen Gemeinde waren, aber später als «Juden» nach den Nürnberger Rassegesetzen definiert wurden, nicht enthalten. In die Liste potentiell jüdischen Eigentums wurden daher alle Häuser mit aufgenommen, in denen nach 1933 ein Eigentumswechsel stattgefunden hat, sowie zusätzlich alle, deren Eigentümer im Ausland wohnten. Abschliessend wurde diese Liste mit zwei weiteren Aufstellungen zu ehemals jüdischen Grundstücken - zusammengestellt von der Magistratsverwaltung (Ostberliner Verwaltung bis 1990) sowie der unmittelbar nach dem Krieg eingesetzten sowjetischen Militäradministration (SMAD) - abgeglichen. Am Ende dieser Recherche stand eine Liste mit ca. 250 Grundstücken vermutlich ehemals jüdischer Eigentümer. Die weitere Arbeit fand mit den historischen Grundbüchern und -akten im Berliner Grundbucharchiv statt. Darin finden sich für jedes Grundstück Schriftstücke zu sämtlichen Eigentumsübergängen seit dem Zeitpunkt des Hausbaus. Im Anschluss an diese Aktenauswertung wurde die Anzahl jüdischer Grundstücke reduziert auf eine Liste mit 112 gesichert jüdischen Grundstücken im Gebiet Helmholtzplatz. Bei insgesamt ca. 600 Grundstücken im Gebiet entspricht das einem Anteil von knapp 20\% in jüdischem Eigentum.

Aus Zeitgründen nicht verwendet wurden die per- sonenbezogenen Quellenbestände zu den jüdischen Eigentümern aus dem Bestand der Oberfinanzdirektion Berlin («Vermögensverwertungsstelle»). Nicht, bzw. noch nicht zugänglich waren die Steuerunterlagen bei den Finanzämtern sowie die Akten des Amtes zur Regelung offener Vermögensfragen, das in Vorbereitung auf die Rückgabe der Grundstücke die Eigentumshistorie aufgearbeitet hat.

\subsection{Jüdisches Immobilieneigentum am Helmholtz- platz}

Fast die Hälfte der untersuchten jüdischen Eigentümer am Helmholtzplatz hatte ihre Immobilien während der Hochphase der Inflation gekauft (vgl. Tab. 2). Lediglich neun Eigentümer waren bereits vor der Inflation Eigentümer der Häuser, sechs davon bereits vor dem Ersten Weltkrieg. Jüdische Eigentümer gehörten also nur vereinzelt der ersten Eigentümergeneration der Berliner Mietshäuser an, die die Häuser kurz nach Erstellung häufig von bauausführenden Handwerkern erworben hatten. Die zweitgrösste Gruppe unter den jüdischen Eigentümern am Helmholtzplatz bildeten diejenigen, die ihre Häuser nach der Währungsstabilisierung kauften. Viele Aufkäufer aus der Inflationszeit verkauften nach der Währungsstabilisierung und der damit verbundenen Wertsteigerung ihre Häuser weiter. Vier Grundstücke wurden noch nach der Machtübernahme durch die Nationalsozialisten am 30.1.1933 von jüdischen Käufern erworben - mit einer Ausnahme allerdings alle bis 1935.

Knapp die Hälfte und damit die grösste Gruppe der jüdischen Eigentümer lebte in Berlin, die Mehrzahl

\begin{tabular}{|l|r|r|}
\hline Zeitpunkt des Erwerbs & Anzahl & $\begin{array}{c}\text { Anteil in } \\
\%\end{array}$ \\
\hline vor 1914 & 6 & 5,3 \\
\hline $1914-1918$ & 0 & 0.0 \\
\hline $1918-1921$ & 3 & 2,7 \\
\hline $1922 / 23$ & 51 & 45,5 \\
\hline $1924-1932$ & 41 & 36,6 \\
\hline ab 1933 & 4 & 3,6 \\
\hline $\begin{array}{l}\text { Erbschaft } \\
\text { Zeitpunkt des ursprünglichen Erwerbs } \\
\text { unbekannt) }\end{array}$ & 3 & 2,7 \\
\hline ohne Angabe & & 3,6 \\
\hline Gesamt & 4 & $\mathbf{1 0 0 . 0}$ \\
\hline
\end{tabular}

Tab. 2: Jüdische Eigentümer im Gebiet Helmholtzplatz: Erwerbszeitpunkt Jewish real estate owners in the Helmholtzplatz area: year of purchase Propriétaires juifs sur le territoire du Helmholtzplatz: année d'acquisition Quelle: Grundakten, Grundbucharchiv Berlin; eigene Berechnungen 


\begin{tabular}{|l|r|r|}
\hline Eigentumsübergänge & Anzahl & Anteil in \% \\
\hline Verkäufe und Zwangsversteigerungen & 14 & \\
\hline Bis 15.September 1935 & 24 & \\
\hline 16. September 1935 - 9. November 1938 & 40 & \\
\hline 9.November 1938 - Ende 1943 & $\mathbf{7 8}$ & \\
\hline Gesamt & & \\
\hline Beschlagnahme, Verfall & 20 & \\
\hline Haupttreuhandstelle Ost (HTO) ab 1940 & 9 & \\
\hline davon weiterverkauft & 3 & \\
\hline 11. Verordnung zum Reichsbürgergesetz 1941 & 3 & \\
\hline Übriger Verfall & 2 & \\
\hline Feindliches Vermögen & 4 & \\
\hline «Arisierungstreuhänder» & $\mathbf{3 2}$ & \\
\hline Gesamt & & \\
\hline Ohne Übergänge & $\mathbf{4}$ & $\mathbf{2 8 . 1}$ \\
\hline Gesamt & $\mathbf{1 1 4}$ & $\mathbf{1 0 0 . 0}$ \\
\hline
\end{tabular}

Tab. 3: Zeitpunkt und Anzahl der «Arisierungen» im Gebiet Helmholztplatz

Date and number of «aryanizations» in the Helmholtzplatz area

Moment et nombre des «aryanisations» sur le territoire du Helmholtzplatz

Quelle: Grundakten, Grundbucharchiv Berlin; eigene Berechnungen

von ihnen in den bürgerlichen Bezirken im Westen der Stadt. Ein gutes Drittel der Eigentümer (40 von 112) lebte zum Zeitpunkt des Kaufes im Ausland, die Hälfte davon in Polen, gefolgt von der Tschechoslowakei und Österreich. Diese Anteile entsprechen in etwa den angeführten Daten der Reichswohnungszählung von $1927 \mathrm{zu}$ ausländischen Investoren in Berlin. Auch eine Erhebung der Steuerämter zu jüdischem Grundeigentum in Berlin von ca. 1938 zeigt Analogien hinsichtlich der Verteilung ausländischen Grundeigentums und der Verteilung der Häuser in jüdischem Eigentum über das Stadtgebiet. Die meisten Grundstücke in jüdischem Eigentum lagen dieser Erfassung zufolge in den westlichen Bezirken Charlottenburg, Wilmersdorf und Schöneberg, sowie in den östlichen Zentrumsbezirken Prenzlauer Berg und Mitte (LAB Pr.Br.Rep 57). Gleichzeitig waren dies auch die Bezirke mit den höchsten Anteilen ausländischen Grundeigentums (vgl. Abb. 1). Die räumlichen Schwerpunkte des jüdischen Grundeigentums entsprachen damit nicht nur den Bezirken mit den höchsten Anteilen von Eigentümern mit ausländischer Staatsangehörigkeit, sondern zudem auch den Hauptwohnorten der jüdischen Berliner (RichaRZ 1988: 180).

Nur in vier Fällen fungierten juristische Personen als Eigentümer, in allen anderen Fällen waren eine oder mehr Privatpersonen als Eigentümer im Grundbuch eingetragen. Allerdings können auch - ähnlich wie heute - die Inhaber oder Teilhaber von Firmen als Privatpersonen und nicht als Gesellschaft im Grundbuch eingetragen gewesen sein. Nicht selten besassen Frauen allein die Häuser, entweder um bei einem selbständig tätigen Ehemann im Falle eines Konkurses die Vollstreckung in das Grundstück zu verhindern, oder die Immobilien dienten bei alleinstehenden Frauen oder Witwen als Unterhalts- bzw. Altersversorgung oder Geldanlage.

Der Anteil selbstnutzender Eigentümer war sehr niedrig. Lediglich 8 der 112 jüdischen Eigentümer lebten selbst in Prenzlauer Berg, die Hälfte davon im eigenen Haus. Oft besassen die Eigentümer aber mehrere Häuser in Berlin und wohnten selbst in dem attraktivst gelegenen Haus, wozu der Helmholtzplatz nicht zwangsläufig zählen muss.

Zur Sozial- und Berufsstruktur der jüdischen Eigentümer sind bedingt durch die Häufung der Berufsnennung «Kaufmann» im Kaufvertrag kaum Aussagen möglich. Die Bandbreite kaufmännischer Tätigkeiten ist so gross - etwa vom kleinen Ladeninhaber bis zum Fabrikbesitzer und Bankdirektor -, dass sich daraus kaum Rückschlüsse ziehen lassen. 


\subsection{Die «Arisierung» des jüdischen Grundeigentums am Helmholtzplatz}

Bis auf vier Grundstücke bzw. Grundstücksteile, bei denen aus den Akten kein Eigentumsübergang ersichtlich ist, wurden alle jüdischen Grundstücke am Helmholtzplatz bis 1945 «arisiert» - etwa zwei Drittel davon über Verkäufe und Versteigerungen, ein Drittel über Beschlagnahme und Verfall an das Deutsche Reich (vgl. Tab.3). Insgesamt ist die Zahl der «Arisierungen» etwas höher als die Anzahl jüdischer Grundstücke, da gelegentlich bei mehreren Eigentümern pro Grundstück mit den einzelnen Grundstücksteilen unterschiedlich verfahren wurde. Auch wenn in vereinzelten Grundbüchern bis 1945 keine Eigentumsübergänge erkennbar sind, heisst das nicht zwangsläufig, dass die Grundstücke noch in jüdischem Eigentum waren, denn die bürokratische Aufarbeitung hinkte dem tatsächlichen «Arisierungs»geschehen erheblich hinterher.

Etwas mehr als zwei Drittel aller jüdischen Grundstücke am Helmholtzplatz wurden im Wege des Verkaufs oder der Versteigerung «arisiert». Nahezu die Hälfte davon wechselte vor dem Novemberpogrom den Eigentümer: 14 Eigentümer haben ihre Häuser bis zum Erlass der Nürnberger Gesetze im September 1935 verkauft, 24 Eigentümer im Zeitraum vom September 1935 bis zum 9.11.1938. Die andere Hälfte der Verkäufe und Versteigerungen am Helmholtzplatz fand nach dem 9.11.1938 statt. Auch am Helmholtzplatz lässt sich somit erkennen, welche Verkaufswelle die Pogromnacht und die unmittelbar darauf folgende drastische Verschärfung der Verfolgungsmassnahmen auslösten.

Das letzte Drittel der Grundstücke in jüdischem Eigentum am Helmholtzplatz wurde beschlagnahmt oder verfiel dem deutschen Reich. Entsprechend dem relativ hohen Anteil polnisch-jüdischer Eigentümer am Helmholtzplatz wurden insgesamt 20 Grundstücke von der HTO beschlagnahmt. Mehr als die Hälfte davon wurde nicht weiterverkauft. Bei diesen Grundstücken ist anzunehmen, dass es sich um solche handelte, die für ganz bestimmte Käufer vorbehalten werden sollten. Drei jüdische Eigentümer am Helmholtzplatz wurden mit der 11. Verordnung zum Reichsbürgergesetz enteignet.

Auch wenn es keinen Erlass zur «Zwangsarisierung» des Grundeigentums gegeben hatte, hiess das nicht, dass es am Ende der NS-Herrschaft noch jüdische Grundeigentümer gegeben hat. Die «Zwangsarisierung» verlief im Fall von Immobilieneigentum lediglich nicht in Form eines Zwangsverkaufs an andere Privateigentümer, sondern in Form von Enteignungen.

\subsection{Die neuen Eigentümer ehemals jüdischer Grundstücke}

Die Gruppe der Aufkäufer ehemals jüdischer Grundstücke (sog. "Ariseure») unterschied sich hinsichtlich sozioprofessioneller Merkmale sowie der Wohnortver- teilung von den jüdischen Voreigentümern. Aus mehreren Akten war weiterhin ersichtlich, dass Kaufinteressenten im Kontakt mit Amts- und Parteistellen ihre nationalsozialistische Weltanschauung betonten, um eine raschere und ihnen wohlwollende Bearbeitung der Anträge zu erwirken (Zunzer 1997: 329).

Knapp zwei Drittel der neuen Eigentümer und damit ein etwas höherer Anteil als bei den Voreigentümern lebten in Berlin selbst. Zum Teil wohnten sie in den bürgerlichen Bezirken im Westen der Stadt, aber auch in den eher kleinbürgerlich und mittelständisch geprägten Bezirken, die als Wohnorte der jüdischen Eigentümer überhaupt nicht oder nur in Einzelfällen vorgekommen waren (Tempelhof, Spandau, Neukölln, Reinickendorf).

Die Berufsangaben in den Kaufverträgen bewegten sich gegenüber denen der jüdischen Voreigentümer in einem breiteren Spektrum. Sowohl der Anteil an Handwerksberufen, aber auch der Anteil der Akademiker hatte zugenommen. Eigentümergemeinschaften, wie sie gelegentlich bei Inflationskäufern aufgetaucht waren, gab es kaum noch, dafür traten mehr Ehepaare als Eigentümer auf. Mehrfacheigentümer kamen, soweit ersichtlich, im Gebiet nicht vor.

Weitergehende Untersuchungen könnten unter Zuhilfenahme umfassenderer sozioökonomischer Indikatoren genaueren Aufschluss darüber liefern, inwiefern die Nationalsozialisten eventuell über die zunehmenden staatlichen und parteilichen Eingriffe in die Zwangsverkäufe sowie über die Verkäufe durch staatliche Stellen selbst eine ganz bestimmte Eigentümerklientel begünstigten und bevorzugt einsetzten. Personenbezogene Quellenbestände könnten darüber hinaus Einblick in Details des Verkaufsgeschehens ermöglichen, wie etwa zur Kaufpreisentwicklung und zu Einzelregelungen der Kaufverträge.

\section{Fazit}

Mit einem sorgfältig orchestrierten Massnahmenbündel stellt die «Arisierung» des Grundeigentums einen Teil der auf die Vernichtung der jüdischen Bevölkerung ausgerichteten Gesamtpolitik des NSRegimes dar. Es wurden rechtliche Regelungen geschaffen, um jüdische Vermögenswerte lückenlos zu dokumentieren und unter Kontrolle zu bringen. Mit diversen Gesetzen und Verordnungen sollte dem Ansinnen der vollständigen Verdrängung der Juden aus allen Wirtschaftsbereichen eine quasirechtsstaatliche Legitimation verliehen werden, hinter der die Akteure ihr Tun verstecken konnten und die zugleich Eingriffe des Auslands vermeiden sollte.

Das NS-Regime bezweckte zweierlei mit der «Arisierung» des Grundeigentums. Zum einen sollten die jüdischen Eigentümer mit einer wachsenden Anzahl 
von Regelungen zunehmend gewaltsam verdrängt werden. Zum anderen wurden die dadurch freigesetzten Vermögenswerte zu einem sukzessive grösser werdenden Teil bis hin zur gesamten Beschlagnahme bzw. Verfallserklärung zugunsten des Deutschen Reiches eingezogen. Durch die Einführung von Preiskontrollen, «Arisierungsabgaben» u.ä.zog das NS-Regime aus diesen erzwungenen Eigentumstransfers einen maximalen finanziellen Profit.

In der lokalen Fallstudie konnte aufgezeigt werden, dass trotz der Tatsache, dass es bis zum Ende der NSHerrschaft keinen Erlass zur zwangsweisen «Arisierung» des jüdischen Grundeigentums gegeben hatte, bis 1945 das gesamte jüdische Immobilieneigentum vernichtet worden war. Auf der Grundlage des lückenlos erfassten jüdischen Vermögens wurden ab Anfang der 40er Jahre die noch in jüdischem Eigentum verbliebenen Grundstücke enteignet. D.h. jüdisches Grundeigentum ging in "arisches» Privateigentum oder in Staatseigentum über. Erst mit der Restitution erhielten in den 90er Jahren die Erben der jüdischen Eigentümer bzw. die Nachfolgeorganisationen für erbenloses jüdisches Vermögen wieder die Verfügung über ihr Eigentum.

Unter stadtgeographischen Gesichtspunkten ist darüber hinaus die Frage interessant, ob die «Arisierung» gleichzeitig eine Zäsur der Eigentümerstruktur darstellt, die evtl. Folgen für die weitere Stadtentwicklung hat. Die "Arisierung» hatte keinen markanten Bruch der Eigentümerstruktur zur Folge. Die Häuser befanden sich auch nach der "Arisierung» weitgehend in privatem Einzeleigentum. Eine deutliche Veränderung der Eigentümerstruktur hat erst nach der Gründung der DDR mit der Überführung in Staatseigentum eingesetzt. Eine Konsequenz der «Arisierung» könnte eher eine subtilere Verschiebung innerhalb der Sozialstruktur der Privateigentümer sein. Die Unterschiede hinsichtlich der Berufsstruktur und der Wohnorte zwischen den jüdischen und den auf die «Arisierung» folgenden Eigentümern deuten auf einen solchen Wandel der Eigentümerstruktur infolge der «Arisierung» hin.

\section{Literatur}

Adler, H.G. (1974): Der verwaltete Mensch. Studien zur Deportation der Juden aus Deutschland. - Tübingen: Verlag unbekannt.

Albrecht, G. et al. (1930): Handwörterbuch des Wohnungswesens. - Jena: Fischer.

BAJOHR, F. (2000): «Arisierung» als gesellschaftlicher Prozess. Verhalten, Strategien und Handlungsräume jüdischer Eigentümer und "arischer» Erwerber. In: Fritz-Bauer-Institut (Hrsg.): «Arisierung» im Nationalsozialismus. Volksgemeinschaft, Raub und
Gedächtnis. - = Jahrbuch 2000 zur Geschichte und Wirkung des Holocaust. - Frankfurt, New York: Campus: 15-30.

BarKaI, A. (1988): Vom Boykott zur «Entjudung». Der wirtschaftliche Existenzkampf der Juden im Dritten Reich 1933-1943. - Frankfurt/Main: Fischer.

BetriffT: «AKtion 3» (1998): Deutsche verwerten jüdische Nachbarn. Dokumente zur Arisierung, ausgewählt und kommentiert von Wolfgang Dressen. Berlin: Aufbau-Verlag.

BRomвACH, H. (1941): Bau- und Reparaturauflagen beim Erwerb städtischer Grundstücke. - In: Deutsche Verwaltung. Organ der Verwaltungsrechtswahrer des nationalsozialistischen Rechtswahrerbundes Jg. 18: 216-217.

DAS GRUNDEIgENTUM:älteste und grösste Zeitschrift für Haus- und Grundbesitz. Herausgeber und Eigentümer: Bund der Berliner Haus- und Grundbesitzervereine e.V.

Deutsche WohnungswirTsChafT:Verkündungsblatt des Zentralverbandes Deutscher Haus- und Grundbesitzervereine e.V. und des Preussischen Landesverbandes der Haus- und Grundbesitzervereine e.V.

EINIGE Dokumente zur Rechtsstellung der Juden und zur Entziehung ihres Vermögens 1933-1945 (1954). = Schriftenreihe zum Berliner Rückerstattungsrecht VII. - Berlin: ohne Verlag.

GensChel, H. (1966): Die Verdrängung der Juden aus der Wirtschaft im Dritten Reich. - Göttingen: Verlag unbekannt.

GLOCK, B.\& K. Keller (2001): Restitution und Stadtentwicklung. Zu den sozialräumlichen Folgen der Restitution in suburbanen und innerstädtischen Gebieten. - In: Deben, L. \& J. Van De Ven (Hrsg.): Berlin und Amsterdam. Globalisierung und Segregation. Amsterdam: Het Spinhuis: 92-108.

Gruner, W. (2000): Die Grundstücke der «Reichsfeinde». Zur "Arisierung» von Immobilien durch Städte und Gemeinden 1938-1945. - In: Fritz-BAuERINSTITUT (Hrsg.): «Arisierung» im Nationalsozialismus. Volksgemeinschaft, Raub und Gedächtnis. - = Jahrbuch 2000 zur Geschichte und Wirkung des Holocaust. - Frankfurt, New York: Campus: 125-156.

HilberG, R. (1990): Die Vernichtung der europäischen Juden. - Frankfurt/Main: Fischer (englische Originalausgabe 1961).

Historikerkommission (Hrsg.); Bailer-Galanda, E. et al. (2000a): "Arisierung» und Rückstellung von Wohnungen in Wien. Die Vertreibung der jüdischen Mieter und Mieterinnen aus ihren Wohnungen und das verhinderte Wohnungsrückstellungsgesetz. - Wien: ohne Verlag.

Historikerkommission (Hrsg.); Graf, G. (2000b): Der Entzug von Mietrechten. Ein rechtshistorischer und rechtsdogmatischer Bericht unter besonderer Berücksichtigung der Entwicklungen nach der Wie- 
dererrichtung der Republik Österreich. - Wien: ohne Verlag.

ReimanN, B. (2000): Städtische Wohnquartiere. Der Einfluss der Eigentümerstruktur. Eine Fallstudie aus Berlin-Prenzlauer Berg. - Opladen: Leske + Budrich.

RICHARZ, M.(1988): 1918-1933. Erfolg und Gefährdung in der Weimarer Republik. - In: Juden in Berlin 1674-1945: ein Lesebuch. - Berlin: Nicolai.

SCHEFfler, W. (1964): Judenverfolgung im Dritten Reich. - Berlin: Büchergilde Gutenberg.

Scholl, A. (1940): Die Entjudung des deutschen Grundbesitzes. - Berlin.

VAN LAAK, D. (1992): Die Mitwirkenden bei der «Arisierung». Dargestellt am Beispiel der rheinischwestfälischen Industrieregion 1933-1940. - In: BüTTNER, U. (Hrsg.): Die Deutschen und die Judenverfolgung im Dritten Reich. - Hamburg: Christians: 232-249.

WALK, J. (Hrsg.) (1981): Das Sonderrecht für die Juden im NS-Staat. Eine Sammlung der gesetzlichen Massnahmen und Richtlinien. Inhalt und Bedeutung. - Heidelberg: Müller.

WISDALSKI, M. (1989): Judenfeindliche Mietgesetze vor fünfzig Jahren. Wohnungsterror unterm Hakenkreuz. - In: Mietermagazin Heft 4: 13-19.

ZunZer, D. (1996): Die «Arisierung» des jüdischen Grundbesitzes - dargestellt am Fallbeispiel Helmholtzplatz Berlin - Prenzlauer Berg. - = HumboldtUniversität zu Berlin, Institut für Geschichtswissenschaften. Unveröffentlichte Magisterarbeit.

ZUNZER, D. (1997): «Die deutsche Geschichte kommt als Gespenst zurück». Die «Arisierung» des jüdischen Hausbesitzes am Helmholtzplatz. - In: Kulturamt Prenzlauer Berg, Prenzlauer Berg Museum für Heimatgeschichte und Stadtkultur (Hrsg.): Leben mit der Erinnerung. Jüdische Geschichte in Prenzlauer Berg. Berlin: Edition Hentrich: 316-332.

\section{Archivalien}

LAB (Landesarchiv Berlin): Pr. Br. Rep. 57: Stadtpräsident der Reichshauptstadt Berlin, Preisbildungsstelle, «Entjudung des Berliner Grundbesitzes».

\section{Zusammenfassung: Die «Arisierung» von jüdischem Grundeigentum während des Nationalsozialismus: ein Fallbeispiel aus Berlin - Prenzlauer Berg}

Nach der Wiedervereinigung der beiden deutschen Staaten im Jahr 1990 wurde in den neuen Bundesländern der weitgehend verstaatlichte Grund und Boden in Privateigentum zurücküberführt. Da in der DDR eine Wiedergutmachung nationalsozialistischen Unrechts weitgehend ausgeblieben war, wurde die Restitutionsregelung auf die Eigentumsentziehungen bis 1933 ausgedehnt.

Die wenig erforschte «Arisierung» des Grundeigentums während des Nationalsozialismus gewann damit an erneuter Aktualität. Die "Arisierung» als Teil der nationalsozialistischen Judenverfolgung bedeutete eine vollständige Verdrängung der Juden aus der Wirtschaft und damit auch aus dem Immobilienbereich. Das Ergebnis der «Arisierung» war «eine der grössten Besitzumschichtungen der Neuzeit».

Ziel des Artikels ist es, einen Überblick über die rechtlichen Regelungen und den Ablauf der «Arisierung» des Grundeigentums zu geben und mit einer Fallstudie diesen Prozess für ein konkretes Quartier im Ostteil Berlins darzustellen.

Summary: The «Aryanization» of Jewish Real Estate Property during National Socialism: a case study in Berlin - Prenzlauer Berg

After German reunification in 1990 , property which had been mostly "people's property» during the GDR, was transferred back to private ownership. In the GDR very little had been done to compensate National Socialist crimes. As a result the restitution law was extended to include property deprivations as far back as 1933.

As a consequence, the "aryanization» of real estate property during National Socialism became once again a relevant and much discussed issue. "Aryanization» as part of the National Socialist persecution of Jewish people resulted in a complete exclusion of the Jewish community from economic activities, as well as from the real estate market. The consequence was «one of the largest ownership transfers in recent times».

This article aims to give an overview of the legal regulations and the way in which the "aryanization» of real estate took place. The process is exemplified by means of a case study of a particular area in the eastern part of Berlin.

\section{Résumé: «L'aryanisation» de la propriété foncière pendant le régime nazi en Allemagne: une étude de} cas relative au quartier du «Prenzlauer Berg» de Berlin Après la réunification des deux Allemagnes la décision fut prise de restituer à ses anciens propriétaires le domaine foncier qui avait été en grande partie nationalisé par suite de la prise de pouvoir du régime socialiste dans l'ex-RDA. Comme celle-ci n'avait pas procédé à la restitution des biens fonciers confisqués par le régime nazi, la nouvelle législation est remontée jusqu'en 1933 en matière de mesures de compensation des dommages causés par le régime nationalsocialiste.

La question de l'«aryanisation» des biens fonciers durant la période national-socialiste gagna de nouveau en intérêt. L' «aryanisation» en tant que composante de la persécution juive par le national-socialisme signifia l'élimination totale des juifs de la vie économique et par là-même aussi du domaine immobilier. Le résultat de cette «aryanisation» fut l'un des plus grands transferts de propriété des temps modernes. Le présent article a pour but de donner un aperçu 
sur les dispositions légales et procédures qui déterminaient l'«aryanisation» de la propriété foncière.

A l'appui de l'étude de cas, il doit illustrer le processus de l'«aryanisation» dans le quartier du «Prenzlauer Berg» à Berlin-Est.

Daniela Zunzer, M.A., Geographisches Institut der Universität Basel, Abteilung Humangeographie / Stadt- und Regionalforschung, Klingelbergstrasse 16, CH-4056 Basel.

e-mail: daniela.zunzer@unibas.ch 